\title{
Canalization and developmental instability of the fetal skull in a mouse model of maternal nutritional stress
}

\author{
Paula N. Gonzalez ${ }^{1,2}$, Federico P. Lotto ${ }^{2}$, and Benedikt Hallgrímsson ${ }^{3}$ \\ ${ }^{1}$ CONICET, Instituto de Genética Veterinaria, Facultad de Ciencias Veterinarias, UNLP-CCT La \\ Plata 1900, Argentina \\ ${ }^{2}$ CONICET, División Antropología, Facultad de Ciencias Naturales y Museo, Universidad \\ Nacional de La Plata, La Plata, Argentina \\ ${ }^{3}$ Department of Cell Biology and Anatomy, University of Calgary, Calgary, AB, Canada
}

\section{Abstract}

Nutritional imbalance is one of the main sources of stress in both extant and extinct human populations. Restricted availability of nutrients is thought to disrupt the buffering mechanisms that contribute to developmental stability and canalization, resulting in increased levels of fluctuating asymmetry (FA) and phenotypic variance among individuals. However, the literature is contradictory in this regard. This study assesses the effect of prenatal nutritional stress on FA and among-individual variance in cranial shape and size using a mouse model of maternal protein restriction. Two sets of landmark coordinates were digitized in three dimensions from skulls of control and protein restricted specimens at E17.5 and E18.5. We found that, by the end of gestation, maternal protein restriction resulted in a significant reduction of skull size. Fluctuating asymmetry in size and shape exceeded the amount of measurement error in all groups, but no significant differences in the magnitude of FA were found between treatments. Convsersely, the pattern of shape asymmetry was affected by the environmental perturbation since the angles between the first eigenvectors extracted from the covariance matrix of shape asymmetric component of protein restricted and control groups were not significantly different from the expected for random vectors. In addition, among-individual variance in cranial shape was significanlty higher in the protein restricted than the control group at E18.5. Overall, the results obtained from a controlled experiment do not support the view of fluctuating asymmetry of cranial structures as a reliable index for inferring nutritional stress in human populations.

\section{Keywords}

protein restriction; fluctuating asymmetry; phenotypic variation; fetal growth

\footnotetext{
Nutritional deprivation is considered as one of the main sources of stress in both extant and extinct human populations with pronounced effects on individual growth and development (Bogin, 1999; Rush, 2000; Ruff et al., 2013). Organisms are particularly susceptible to
}

Corresponding author: Paula N. Gonzalez, Tel. 221 421-1799, Fax. 421-1799, paulan.gonza@gmail.com, pgonzalez@fcnym.unlp.edu.ar. 
environmental insults during key ontogenetic periods, especially during prenatal growth, when morphogenesis is taking place and the rate of cell replication and differentiation is high. Accordingly, an extensive body of knowledge shows that unfavorable intrauterine environments produce a coordinated set of responses which may cause permanent physiological and structural changes with later consequences for individual life (Gluckman et al., 2005, 2007; Low et al., 2012). This is mediated by the capacity of developmental systems to produce different phenotypes in response to environmental changes, an extended property of organisms known as phenotypic plasticity (Pigliucci, 2001; Fusco and Minelli, 2010). In addition, early exposure to adverse influences such as unbalanced nutrition or limited availability of oxygen is thought to disrupt the buffering mechanisms (molecular chaperones, gene redundancy, etc.; Willmore and Hallgrímsson, 2005) that contribute to the robustness of phenotypes against internal and external perturbations (Parsons, 1992; Leung et al., 2000; de Visser et al., 2003; reviewed in Hallgrímsson, 1998). These mechanisms account for the ability of organisms to develop a constant phenotype despite genetic or environmental variability (canalization) as well as for the tendency for development to follow the same trajectory under specific genetic and environmental conditions (developmental stability) (Waddington, 1942, Van Valen, 1962; Leamy and Kingenberg, 2005; Willmore et al., 2007). The alteration of buffering capacity of developmental systems will result in the accumulation of small perturbations originated by the sum of stochastic events affecting development. As a consequence, an increase of variation among traits within individuals as well as between individuals is expected (Palmer, 1994; Leung et al., 2000; Hoffman and Woods, 2003; Van Dongen and Gangestad, 2011).

A commonly held view is that when resources are limited, their allocation is crucial for organism survival and thus nutritional restriction will significanlty reduce the amount of energy spent in mechanisms that repair errors originated during organ morphogensis. Consequently, the magnitude of phenotypic variance in populations with limited access to nutrients will be augmented. These phenotypic responses to environmental stress have been of great interest because of their potential use to make inferences about human populations in the past (De Leon, 2007; Gawlikowska et al., 2007; Hoover and Matsumara, 2008; Bigoni et al., 2013). Nevertheless, the available evidence supporting an association between stress and indirect measures of developmental stability and canalization based on phenotypic variance is inconsistent (Kujanová et al., 2008; Özener, 2010; Jeong et al., 2013). This might be partially due to the fact that most studies are based on comparative data where other confounding factors are poorly controlled. In this sense, animal models, for which different sources of variation can be manipulated, are fundamental for understanding the effect of specific environmental factors on phenotypic variability (Hallgrímsson and Lieberman, 2008). However, only a few experiments in controlled settings have evaluated the influence of prenatal stress on robustness of morphological traits and their results have been mixed (Mooney et al., 1985; Kohn and Bennett, 1986; Jones and German, 2005). Some studies that measure the magnitude of small, random deviations of both sides of bilateral symmetric structures (i.e., fluctuating asymmetry, FA) support an increase of intra-individual variation of dental, long bone and parietal variables of neonatal rats with exposure to prenatal temperature and noise stress, and early nutrient restriction (Sciulli et al., 1979; Mooney et al., 1985). Others show that low-protein diets in rats negatively impact bone growth and 
increase variation in ontogenetic trajectories among individuals but have no effect on intraindividual variation (Jones and German, 2005).

The focus of most studies aimed at analyzing the effect of external factors on developmental buffering has been assessing the magnitude of the between and within groups variances as a measure of stress. Because the methodological approach used to quantify morphological traits was mainly based on linear measures, the influence of developmental perturbations on the spatial patterns of morphological variation have not been extensively explored.

Geometric morphometric methods that incorporate and preserve the spatial relations of variables into multivariate analyses were shown to be a powerful tool to detect subtle differences both in the amount and pattern of morphological variation in the context of investigating developmental stability and canalization (Klingenberg and McIntyre, 1998; Mardia et al., 2000; Palmer et al., 2010). This methodological approach has been used to discuss whether differences in the magnitude of asymmetry related to genetic and environmental factors are caused by an amplification of the same developmental processes or different processes are involved (Debat et al., 2011). Under the hypothesis of shared mechanisms, the spatial patterns of shape asymmetry are expected to be invariable. However, this has been tested only on a few structures and organisms, and thus the conclusions drawn from them are rather limited.

The aim of this study was to assess the influence of prenatal nutritional stress on the developmental buffering of cranial traits under controlled conditions by using geometric morphometric techniques to quantify shape variation. In order to control for other confounding factors we used a mouse model of maternal protein restriction during pregnancy. Cranial variation was assessed at E17.5 and E18.5 post-conception days by means of coordinates of anatomical landmarks in 3D. Specifically, we tested the following hypotheses: 1) maternal malnutrition has a negative impact on DS and canalization of the fetal skull. Particularly, the protein restricted group is expected to depict an increase in the magnitude of intra and inter individual variation of skull size and shape, measured by the amount of fluctuating asymmetry and phenotypic variation around the group mean, respectively; and 2) early malnutrition affects the pattern of shape asymmetry of the skull. Differences in principal components based on the shape asymmetry matrices for control and nutrient restricted groups are expected if the pattern of shape asymmetry is changed by this environmental perturbation.

\section{Material and methods}

C57BL/6J male and female 4-wk-old mice were purchased from The Jackson Laboratory (Bar Harbor, ME) and maintained on a 12-h light/12-h dark cycle for 4 weeks. During this period of acclimatization they were fed on a control diet (20\% protein TD91352, Harlan Teklad, Madison). Then, and two weeks before mating, nulliparous females were randomly divided into two groups, Control (CG) and Low Protein (LPG). Mice in LPG had ad libitum access to a diet with $6 \%$ protein (TD90016, Harlan Teklad, Madison,), while mice in the Control Group (CG) had ad libitum access to the control diet (20\% protein). Both diets are isocaloric $(3.8 \mathrm{Kcal} / \mathrm{g})$ but differ in the amount of protein in the form of casein and DLMethionine. After the two weeks of acclimatization, breeders were placed together overnight 
and females were checked daily for vaginal plugs. When a plug was detected, the female was separated and maintained with the previously assigned diet until the end of the experiment. At 17.5 and 18.5 days post-conception, pregnant dams were sacrificed by cervical dislocation. The uterus was opened to expose the conceptuses, which were removed by drawing a fine pair of forceps between conceptus and uterus. Finally, the sample was composed as follows: (a) Control Group E17.5, $\mathrm{n}=33$; (b) Low Protein Group E17.5, $\mathrm{n}=30$; (c) Control Group E18.5, n=40; (d) Low Protein Group E18.5, n=31.

All animal procedures were reviewed and approved by the University of Calgary Health Sciences Animal Care Committee and experiments were conducted in compliance with this approval. Animals were cared for as per the guidelines of the Canada Council on Animal Care.

The skulls were fixed overnight in $4 \%$ phosphate buffered formaldehyde (PFA) and then micro-computed tomography images ( $\mu$ CT 35, Scanco Medical AG, Brüttisellen, Switzerland) were obtained for both control and restricted protein groups. Forty-two landmarks were digitized on the $\mu \mathrm{CT}$ scans of each mouse cranium using Amira 5.2.2 (Visualization Science Group). Figure 1 shows the bilateral 3D landmarks digitized from the $3 \mathrm{D}$ reconstructions (a detailed definition of the landmarks used is given in Table 1 of supplementary material).

\section{Statistical analyses}

The coordinates of landmarks were aligned by means of a generalized least-squares Procrustes fit. This procedure optimally translates, rotates and scales coordinates of landmarks in order to remove the information on position, orientation and size (Rohlf and Slice, 1990). The Procrustes superimposition was made in MorphoJ 1.05c (Klingenberg, 2011). The centroid size of each configuration of points (i.e., the square root of the summed squared distances from all landmarks to the configuration centroid) was estimated for each data set and then used to scale the raw coordinates in the Procrustes superimposition (Bookstein, 1991).

The magnitude of size FA within groups was assessed by computing separately the centroid size of the left and right sides of the skull. Firstly, the signed difference between sides (L-R) was estimated for each skull. The normality of L-R distribution was tested and the outliers were eliminated prior any subsequent analysis. Then, a standard two-way mixed model ANOVA was used to assess the significance of FA (individual:side) and directional asymmetry (DA, side effect) within each sample (Palmer 1994). When repeated measures are taken, this test evaluates whether FA is larger than measurement error. If the presence of significant directional asymmetry was detected, a correction was used to estimate FA without the confounding effects of DA. The formula used was: FAcs $=|(\mathrm{L}-\mathrm{R})-\mathrm{DA}|$. The approach followed here defines FA as the random variation about the mean (L-R) of a given bilateral trait, regardless of whether or not the mean is zero (Graham et al., 1993; Hutchinson and Cheverud, 1995; DeLeon, 2007).

Estimations of FA corrected for ME and directional asymmetry were then derived from these ANOVAs. The FA10 index, according to the terminology by Palmer and Strobeck 
(2003), was estimated as 0.798 (MSsj - MSm), where MSsj is side:individual interaction MS and MSm is the measurement error MS obtained from the ANOVA tables.

In order to assess FA in shape, Procrustes ANOVAs were conducted for each group to simultaneously test the effect of side, individual and the interaction individual:side on the shape variables (Klingenberg and McIntyre, 1998). If the effect of side was significant, DA was corrected by subtracting sample mean values of the difference between sides for each coordinate (i.e. directional asymmetry vector) from the specimen values of the difference between sides for each coordinate (i.e. asymmetric component of shape coordinates). The resulting differences were then squared and summed across all landmarks. The square root of this sum was used as a measure of individual shape FA (called Procrustes FA) following the approach proposed by Klingenberg and McIntyre (1998).

Permutation ANOVA tests were used to evaluate the effect of protein restriction on size and shape FA. For shape data, the Procrustes FA was used as the response variable and treatment as the fixed factor. Differences between treatments in size fluctuating asymmetry was tested using the measure of FA corrected by directional asymmetry. In this model FAcs was the dependent or response variable, while treatment was used as the fixed factor. The ANOVA test was estimated using the function aovp from the lmPerm package (Wheeler, 2010).

The comparison of Procrustes FA between groups quantifies differences in the magnitude of overall asymmetry, but the spatial patterns of asymmetry might be also altered by environmental perturbations. In order to investigate the patterns of shape asymmetry we first computed the covariance matrix based on the asymmetric component, for each age and treatment using the average of the two series of measurements. Then, we performed a Principal Component Analysis on this matrix. The similitude of shape FA patterns among treatments was tested by estimating the angles formed by pairs of eigenvectors from the covariance matrices and comparing the values obtained to expected angles between pairs of random vectors. This allowed to test whether the patterns of FA among groups were more similar than expected from random variation. Lower p-values indicate that the angle between the vectors of interest is significantly different from the expected by chance alone. This procedure is based on Klingenberg and McIntyre (1998) and Debat et al. (2001). We also estimated the angle between the PC1 based on the covariance matrix of the symmetric component in order to compare the effect of treatment on both components of shape variation. The symmetric component of configurations of landmarks with object symmetry is extracted by reflecting each configuration and relabelling the paired landmarks so they match with their counterpart. Then, the original and mirrored configurations are superimposed together by means of a generalized least-squares Procrustes fit. The average of the original and mirrored configuration of each specimen estimated after the overall superimposition represents the symmetric component of shape.

Finally, the amount of phenotypic variation within groups was estimated using the symmetric component of shape variation (Klingenberg et al., 2002; Mardia et al., 2000). The consensus of each group of specimens obtained after the superimposition is also symmetric, and thus the distances of specimen averages with respect to their group mean is a measure of variation in shape. The distance from each specimen to the consensus of its group was 
calculated as the root of square-sum of differences between specimens coordinates and the consensus shape. To test whether the amount of variation differed among groups, we computed a permutation ANOVA of the individual deviations from the respective group averages, using age and treatment as the independent variables.

Both components of shape variation (symmetric and asymmetric) as well as centroid size were obtained in MorphoJ 1.05c (Klingenberg, 2011). This software was also used to calculate the Procrustes ANOVA and compare the angles between eigenvectors of the covariance matrices of the symmetric and asymmetric components of shape. All other analyses were performed using the base and $1 \mathrm{mPerm}$ packages for R (R Core Team 2012).

\section{Results}

\section{a. Size}

Protein restriction in maternal diet resulted in fetal growth impairment, which affected cranial growth. At E17.5, skull size was $2.5 \%$ smaller in the protein restricted than in the Control group, although differences were not significant (Fig. 2). Size decrease was more noticeable at E18.5, where skulls were 5\% and significantly smaller in the restricted group (Fig. 2). Differences in cranial size due to age and treatment, as well as the interaction factor age:treatment were highly significant $(\mathrm{P}<0.05$ with 5000 iterations $)$.

Two-factor ANOVA tests of skull centroid size revealed a significant effect of the interaction individual by side, indicating that FA in size was detectable for every age and treatment (Table 1). Although the percentage of total variation accounted by FA was small (less than 1\%), it was 10 to 20 fold higher than measurement sum-of-squares error. In addition, the effect of side (representing DA) was significantly different from zero at E17.5 and E18.5, in both protein restricted and control groups. It is noteworthy, however, that the amount of variance explained by differences between left and right sides were 2 to 20 fold lower than the variance explained by FA. The largest source of variability in skull size was represented by among-individual differences, but this effect is of no interest in this study. Overall, these results indicated that measurement error can be neglected as a confounding factor in the estimation of FA, while the effect of DA needs to be considered prior the comparison of FA among groups.

The signed L-R mean was positive in the four groups, indicating that the left side was in average larger than the right side. FA in centroid size, after correcting by DA was higher in the restricted protein groups at both ages, although the differences were not significant neither at $\mathrm{E} 17.5\left(F_{1,62}=1.186, \mathrm{P}=0.280\right)$ nor at $\mathrm{E} 18.5\left(F_{1,65}=0.423 ; \mathrm{P}=0.518\right)$. Similar results were obtained using the estimators of FA corrected for ME and directional asymmetry: at both ages the restricted protein group had slightly higher FA10 values than control groups (Table 2).

Overall centroid size of each skull (estimated using all paired landmarks) was plotted versus the FAcs to evaluate whether or not FA is associated with size. No relation was found between these variables, indicating that the degree of asymmetry was not size-dependent (results not shown). 


\section{b. Shape}

Concerning skull shape, Procrustes ANOVA performed on the superimposed coordinates of landmarks allowed the partitioning of shape variation into different sources (Table 3 ). The effect of interaction individual by side was significant in the four groups analyzed and accounted for 20 to $30 \%$ of total variation in skull shape (Table 3). The variance due to measurement error ( 2 to $4 \%$ ) was around 10 fold lower than FA, and thus it was negligible relative to the asymmetric shape variation. The side-effect was also significant, suggesting that the skulls displayed a noticeable DA in shape, although this factor accounted for less than $3 \%$ of total variation in shape (Table 3 ). The results obtained showed that FA is a significant source of variation in fetal skulls.

Due to the significant effect of side in this dataset, the sample directional asymmetry vector was subtracted from individual asymmetric component of shape coordinates. The Procrustes FA was thus estimated on the basis of the corrected coordinates. Correlations between Procrustes FA scores and skull CS were negative and non-significant for both corrected and non-corrected by DA data $(r=-0.191, r=-0.205)$.

With regards to the effect of protein restriction on intra-individual variance, Procrustes FA values grouped by age and treatment were very similar (Fig. 3). Results of the ANOVA test with permutation performed on Procrustes FA scores showed no significant effect of age or treatment on the magnitude of fluctuating asymmetry. The interaction term "treatment:age" was not significant either. Conversely, there are noticeable differences in the amount of phenotypic variation among groups as measured by the Procrustes distance of each specimen to its group mean (Fig. 4). At E17.5 both control and low protein groups display similar values, but at E18.5 the variance of the control group drops while in the low protein group remains as high as at the earlier stage. The ANOVA test with permutations confirmed this result, showing a significant effect of age on the Procrustes distances to the group consensus $(\mathrm{P}<0.05,5000$ iterations $)$.

The comparison of angles between the first asymmetric PC from control and LP groups shows that the values do not differ from expected values for pairs of random vectors (Table 3). Consequently, patterns of shape asymmetry were modified by the environmental perturbation induced even though this factor did not have a significant effect on the magnitude of FA. Conversely, the pattern of variation of the symmetric component was similar between control and treated groups, as is shown by the low and significant values of the angles between PCs1 (Table 4). In order to visually compare the patterns of shape FA among treatments we display the pattern of shape change associated with the first PC estimated from the covariance matrices of asymmetric component (Figure 5). In addition, the distribution of eigenvalues for each group is shown in order evaluate whether shape FA is concentrated or evenly distributed over many directions. Although actual values are not comparable (Debat et al. 2011), the overall shape of eigenvalue distribution was similar in the four groups and showed that FA variation was mostly concentrated in the first 5 PCs. 


\section{Discussion}

This study shows a significant effect of protein restriction during pregnancy on cranial growth, which results, by the end of gestation, in a substantial reduction of skull size. We hypothesized that this environmental perturbation will also increase the magnitude of FA given that DI is expected to increase under stressful conditions. Fetal skulls displayed significant levels of fluctuating asymmetry in size and shape for both control and protein restricted animals, however, no significant differences in the magnitude of FA were found between groups. As discussed below, the failure to detect an effect of prenatal malnutrition on cranial FA is not likely a result of methodological issues. Previous studies vary widely in whether they find a link between stress and FA of morphological traits and so the literature is contradictory in this regard (Siegel and Doyle, 1975, Siegel et al., 1977; Sciulli et al., 1979; Markow, 1995; Woods et al., 1999; Graham et al., 2000; Stige et al., 2004).

Discrepancies among reports may be due to the effect of multiple factors tested temperature, nutrition, radiation, density, noise, etc.- on development, variation in response to stress among species, the intensity of stress used in the study and the ontogenetic period examined.

In this study, animals were exposed to a significant reduction in nutrient supply during a critical period of growth. One possible explanation for our finding that FA did not increase with stress is that errors in development accumulate along ontogeny (Hallgrímsson, 1998, 1999) and thus, differences in FA between undernourished and control groups will arise later in ontogeny. However, similar findings were attained in a previous study where we showed that exposing rats to food restriction from birth to adulthood did not increase the amount of FA of the skull (Gonzalez et al., 2012). Jones and German (2005) also found that groups of rats fed on protein restricted and control diets from weaning to adulthood displayed a relative lack of differences in the individual variation around the growth curves fitted to longitudinal data of each specimen. These results suggest that individual growth trajectories are robust to external perturbation. Another alternative is that under nutritional stress, resource allocation affects somatic growth but not the mechanisms responsible for developmental buffering, which consume around $2 \%$ of a cell's energy (Scriver, 2002). To the best of our knowledge no study has estimated how these mechanisms would be affected by nutrient deprivation. Additionally, the lack of association between FA and stress might be due to strong selection for symmetry which has reduced its sensitivity to stress (Van Dongen et al., 2009). This hypothesis has been claimed to explain why several developmental disorders that result in major morphological abnormalities in human fetuses have no effect on limb FA while others result only in a moderate increase in limb FA (Van Dongen et al., 2009; Wijnaendts et al., 2010).

Contrary to what was observed for the magnitude of FA, at E18.5 the amount of amongindividual variation in the cranial shape was higher in the low protein group than in control animals. Comparable results were obtained in a sample of rats exposed to early calorie restriction, which displayed higher variation in size and shape than the animals fed ad libitum on a regular diet (Gonzalez et al., 2011). In the same way, prolonged protein restriction was shown to increase the variation among individual growth trajectories (Jones and German, 2005). These findings suggest that the effects of environmental perturbations 
on within-group variation and FA, and thus on canalization and developmental stability, might be relatively independent. Even when organisms have been selected for high robustness against developmental noise, their differential susceptibility to environmental perturbations might remain. Consequently, measures of phenotypic variation among individuals may be taken as valid and independent estimations of the buffering capacity of development against external factors. However, one of the main shortcomings of using this approach with natural populations is the confounding effect of microevolutionary processes such as gene flow, migration and genetic drift, which can also affect the levels of phenotypic variation within groups.

Because the power of statistical tests to detect differences in FA among groups is highly affected by both measurement error and directional asymmetry, the influence of these factors in the results obtained here needs to be taken into account. For both components of morphological variation -size and shape-, we found that FA exceeded the amount of measurement error. In addition, significant differences in mean size and shape of left and right sides of the skull were found, although directional asymmetry accounted for a very low proportion of total variation. Despite the similarities between the results obtained for CS and shape, there were also remarkable differences, particularly in the amount of total variance explained by FA. While only $1 \%$ of total variability in size was due to FA, up to $30 \%$ of shape variation was explained by FA. Skull shape was described using a set of coordinates of landmarks, which allows describing the overall asymmetry in a single analysis. As the asymmetry across traits is summed, the asymmetric component of total variation is increased. This is of great importance when several samples are compared because the chances of finding significant differences are strongly correlated with the proportion of FA compared to ME. Van Dongen (1999) demonstrated that when FA largely exceeds ME, two repeated measurements on a sample of 20 individuals have a $100 \%$ power of detecting FA in a single sample. In addition, previous simulation studies have shown that combining multiple univariate descriptors or using shape as FA estimators increases the possibilities of detecting a relationship between developmental instability and FA (Leung et al., 2000; Palmer et al., 2010). Overall, our study supports the view that multivariate descriptors of morphological traits are preferred in order to use FA as a measure of the degree of developmental instability among individuals exposed to different genetic and environmental factors.

One interesting finding that deserves further examination is that the environmental perturbation induced changes in the spatial pattern of FA of the skull while its magnitude was not affected. The relationship between the degree and the pattern of asymmetry in morphological traits has not been extensively studied yet, partially due to some limitations of traditional morphometric techniques for analyzing spatial patterns of shape change (Adams et al., 2004). It has been suggested that increments in the magnitude of FA in Drosophila wings are caused by an amplification of the same developmental processes as the spatial patterns of shape asymmetry remains unchanged (Debat et al., 2011). Our results point out that the processes underlying developmental instability might be altered by environmental perturbations, resulting in changes in the patterns of FA, and simultaneously the magnitude of overall asymmetry remains constant. 
In sum, the evidence discussed in this work show a lack of association between early nutritional stress and an increase in the magnitude of fluctuating asymmetry in cranial shape and size. Even when multiple traits were analyzed jointly and the amount of FA exceeded the measurement error by a large and significant margin, the specimens under severe protein reduction did not display higher levels of FA compared to the control group. This is in agreement with some of the previous experiments on other organisms that also failed to find higher levels of FA in phenotypic traits under conditions of nutritional stress (Stige et al., 2004; Vishalakshi and Singh, 2007; Vijendravarma et al., 2011). Although findings in animal models have to be taken with caution because they are difficult to extrapolate to other species, similarities in developmental structure between human and rodent skulls (Martinez-Abadias et al., 2012) validate the inferences made from this study regarding the effect of environmental factors on fluctuating asymmetry in human populations. Overall, the results obtained here from a controlled experiment do not support the view of fluctuating asymmetry of cranial structures as a reliable index for inferring stress in past human populations.

\section{Supplementary Material}

Refer to Web version on PubMed Central for supplementary material.

\section{Acknowledgments}

This study was supported by the American Association of Physical Anthropologists Professional Development Program, the CIHR Training Program in Genetics, Child Development and Health (Alberta Children's Hospital) and grants from Alberta Innovates - Health Solutions.

\section{References}

Adams DC, Rohlf FJ, Slice DE. Geometric morphometrics: ten years of progress following the "revolution. Ital J Zool. 2004; 71:5-16.

Bigoni L, Krajíček V, Sládek V, Velemínský P, Velemínská J. Skull shape asymmetry and the socioeconomic structure of an early medieval central european society. Am J Phys Anthropol. 2013; 150:349-364. [PubMed: 23283725]

Bogin, B. Patterns of human growth. 2. Cambridge: Cambridge University Press; 1999.

Debat V, Bloyer S, Faradji F, Gidaszewski N, Navarro N, Orozco-ter Wengel P, Ribeiro V, Schlötterer C, Deutsch JS, et al. Developmental stability: A major role for Cyclin G in Drosophila melanogaster. PLoS Genet. 2011; 7:e1002314. [PubMed: 21998598]

DeLeon VB. Fluctuating asymmetry and stress in a medieval Nubian population. Am J Phys Anthropol. 2007; 132:520-534. [PubMed: 17243154]

De Visser JAGM, Hermisson J, Wagner GP, Ancel Meyers L, Bagheri-Chaichian H, Blanchard JL, Chao L, Cheverud JM, Elena SF, et al. Perspective: Evolution and detection of genetic robustness. Evolution. 2003; 57:1959-1972. [PubMed: 14575319]

Fusco G, Minelli A. Phenotypic plasticity in development and evolution: facts and concepts. Phil Trans R Soc B. 2010; 365:547-556. [PubMed: 20083631]

Gawlikowska A, Szczurowski J, Czerwiński F, Miklaszewska D, Adamiec E, Dzieciołowska E. The fluctuating asymmetry of medieval and modern human skulls. Homo. 2007; 58:159-172. [PubMed: 17445814]

Gluckman PD, Hanson MA, Spencer HG, Bateson P. Environmental influences during development and their later consequences for health and disease: implications for the interpretation of empirical studies. Proc Biol Sci. 2005; 272:671-677. [PubMed: 15870029] 
Gluckman PD, Hanson MA, Beedle AS. Early life events and their consequences for later disease: a life history and evolutionary perspective. Am J Hum Biol. 2007; 19:1-19. [PubMed: 17160980]

Gonzalez PN, Oyhenart E, Hallgrimsson B. Effects of environmental perturbations during postnatal development on the phenotypic integration of the skull. J Exp Zool B Mol Dev Evol. 2011; 316:547-61. [PubMed: 21826787]

Gonzalez PN, Oyhenart E, Hallgrimsson B. Nutritional stress and the pattern of fluctuating asymmetry in the skull. Am J Phys Anthropol. 2012; 147(S54):153.

Graham JH, Freeman DC, Emlen JM. Antisymmetry, directional asymmetry, and dynamic morphogenesis. Genetica. 1993; 89:121-137.

Graham JH, Roe KE, West TB. Effects of lead and benzene on the developmental stability of Drosophila melanogaster. Ecotoxicology. 1993; 2:185-195. [PubMed: 24201580]

Hallgrímsson B. Fluctuating asymmetry in the mammalian skeleton - Evolutionary and develop-mental implications. Evol Biol. 1998; 30:187-251.

Hallgrímsson B. Ontogenetic patterning of skeletal fluctuating asymmetry in rhesus macaques and humans: Evolutionary and developmental implications. Int J Primatol. 1999; 20:121-151.

Hallgrímsson B, Lieberman DE. Mouse models and the evolutionary developmental biology of the skull. Integr Comp Biol. 2008; 48:373-384. [PubMed: 21669799]

Hoffmann, AA.; Woods, RE. Associating environmental stress with developmental stability: problems and patterns. In: Polak, M., editor. Developmental instability: causes and consequences. Oxford University Press; 2003. p. 387-401.

Hoover KC, Matsumura H. Temporal variation and interaction between nutritional and developmental instability in prehistoric Japanese populations. Am J Phys Anthropol. 2008; 137:469-478. [PubMed: 18711732]

Hutchinson DW, Cheverud JM. Fluctuating asymmetry in Tamarin (Saguinus) cranial morphology: Intra- and interspecific comparisons between taxa with varying levels of genetic heterozygosity. J Hered. 1995; 86:280-288. [PubMed: 7657996]

Jeong Y, Woo EJ, Pak S. No significant difference in the levels of dental fluctuating asymmetry between hypoplastic and non-hypoplastic skeletal groups from the Joseon Dynasty (mid 15th-early 20th century), South Korea. Arch Oral Biol. 2013; 58:1047-1056. [PubMed: 23528063]

Jones, DC.; German, RZ. Chapter 5 - Variation in Ontogeny. In: Hallgrímsson, B.; Hall, BK., editors. Variation. Burlington: Academic Press; 2005. p. 71-85.

Klingenberg CP, McIntyre GS. Geometric morphometrics of developmental instability: analyzing patterns of fluctuating asymmetry with Procrustes methods. Evolution. 1998; 52:1363-1375.

Klingenberg CP, Barluenga M, Meyer A. Shape analysis of symmetric structures: quantifying variation among individuals and asymmetry. Evolution. 2002; 56:1909-1920. [PubMed: 12449478]

Klingenberg CP. MorphoJ: an integrated software package for geometric morphometrics. Molecular Ecology Resources. 2011; 11:353-357. [PubMed: 21429143]

Kohn LA, Bennett KA. Fluctuating asymmetry in fetuses of diabetic rhesus macaques. Am J Phys Anthropol. 1986; 71:477-483. [PubMed: 3812662]

Kujanová M, Bigoni L, Velemínská J, Velemínský P. Limb bones asymmetry and stress in medieval and recent populations of Central Europe. Int J Osteoarchaeol. 2008; 18:476-491.

Leamy LJ, Klingenberg CP. The genetics and evolution of fluctuating asymmetry. Annu Rev Ecol Evol Syst. 2005; 36:1-21.

Leung B, Forbes MR, Houle D. Fluctuating asymmetry as a bioindicator of stress: Comparing efficacy of analyses involving multiple traits. Am Nat. 2000; 155:101-115. [PubMed: 10657180]

Low FM, Gluckman PD, Hanson MA. Developmental plasticity, epigenetics and human health. Evol Biol. 2012; 39:650-665.

Mardia KV, Bookstein FL, Moreton IJ. Statistical assessment of bilateral symmetry of shapes. Biometrika. 2000; 87:285-300.

Markow TA. Evolutionary ecology and developmental instability. Annu Rev Entomol. 1995; 40:105120. 
Martínez-Abadías N, Mitteroecker P, Parsons TE, Esparza M, Sjøvold T, Rolian C, Richtsmeier JT, Hallgrímsson B. The Developmental Basis of Quantitative Craniofacial Variation in Humans and Mice. Evol Biol. 2012; 39:554-567. [PubMed: 23226904]

Mooney MP, Siegel MI, Gest TR. Prenatal stress and increased fluctuating asymmetry in the parietal bones of neonatal rats. Am J Phys Anthropol. 1985; 68:131-134. [PubMed: 4061598]

Özener B. Fluctuating and directional asymmetry in young human males: Effect of heavy working condition and socioeconomic status. Am J Phys Anthropol. 2010; 143:112-120. [PubMed: 20734438]

Palmer, AR. Fluctuating asymmetry analyses: a primer. In: Markow, TA., editor. Developmental instability: its origins and evolutionary implications. Dordrecht, The Netherlands: Kluwer Academic Publishers; 1994. p. 355-364.

Palmer M, Linde M, Morales-Nin B. Disentangling fluctuating asymmetry from otolith shape. Mar Ecol-Prog Ser. 2010; 399:261-272.

Parsons PA. Fluctuating asymmetry: a biological monitor of environmental and genomic stress. Heredity. 1992; 68:361-364. [PubMed: 1563968]

Pigliucci, M. Phenotypic Plasticity: Beyond Nature and Nurture. JHU Press; 2001.

R Core Team. R: A language and environment for statistical computing. R Foundation for Statistical Computing; Vienna, Austria: 2012. http://www.R-project.org/

Ruff CB, Garofalo E, Holmes MA. Interpreting skeletal growth in the past from a functional and physiological perspective. Am J Phys Anthropol. 2013; 150:29-37. [PubMed: 23283662]

Rush D. Nutrition and maternal mortality in the developing world. Am J Clin Nutr. 2000; 72:212s240s. [PubMed: 10871588]

Sciulli PW, Doyle WJ, Kelley C, Siegel P, Siegel MI, Sciulli PW, Doyle WJ, Kelley C, Siegel P, et al. Interaction of stressors in the induction of increased levels of fluctuating asymmetry in laboratory rats. Am J Phys Anthropol. 1979; 50:279-284. [PubMed: 443361]

Scriver CR. Does hereditary metabolic disease modulate senescence and aging? J Inherit Metab Dis. 2002; 25:235-51. [PubMed: 12137233]

Siegel MI, Doyle WJ. The differential effects of prenatal and postnatal audiogenic stress on fluctuating dental asymmetry. J Exp Zool. 1975; 191:211-214. [PubMed: 1113068]

Siegel MI, Doyle WJ, Kelly C. Heat stress, fluctuating asymmetry and prenatal selection in the laboratory rat. Am J Phys Anthropol. 1977; 46:121-126. [PubMed: 556888]

Stige LC, Hessen DO, Vøllestad LA. Severe food stress has no detectable impact on developmental instability in Daphnia magna. Oikos. 2004; 107:519-530.

Van Dongen SV. Accuracy and power in fluctuating asymmetry studies: effects of sample size and number of within-subject repeats. J Evol Biol. 1999; 12:547-550.

Van Dongen S, Wijnaendts LCD, Ten Broek CMA, Galis F. Fluctuating asymmetry does not consistently reflect severe developmental disorders in human fetuses. Evolution. 2009; 63:18321844. [PubMed: 19245674]

Valen LV. A Study of Fluctuating Asymmetry. Evolution. 1962; 16:125.

Van Dongen S, Gangestad SW. Human fluctuating asymmetry in relation to health and quality: a meta-analysis. Evol Hum Behav. 2011; 32:380-398.

Vijendravarma RK, Narasimha S, Kawecki TJ. Adaptation to larval malnutrition does not affect fluctuating asymmetry in Drosophila melanogaster. Biol J Linn Soc. 2011; 104:19-28.

Vishalakshi C, Singh BN. Effect of environmental stress on fluctuating asymmetry in certain morphological traits in Drosophila ananassae: nutrition and larval crowding. Can J Zool. 2007; $86: 427-437$.

Waddington CH. The epigenotype. Endeavour. 1942; 1:18-20.

Wheeler, RE. multResp() lmPerm. The R project for statistical computing. 2010. http://www.rproject.org/

Wijnaendts L, Galis F, Van Dongen S, Ten Broek C. Human fetuses and limb asymmetry: No evidence for directional asymmetry and support for fluctuating asymmetry as a measure of developmental instability. Animal Biology. 2010; 60:169-182. 
Willmore, KE.; Hallgrímsson, B. Within individual variation: Developmental noise versus developmental stability. In: Hallgrímsson, B.; Hall, BK., editors. Variation. Burlington: Academic Press; 2005. p. 191-218.

Willmore KE, Young NM, Richtsmeier JT. Phenotypic Variability: Its Components, Measurement and Underlying Developmental Processes. Evol Biol. 2007; 34:99-120.

Woods RE, Sgro CM, Hercus MJ, Hoffmann AA. The association between fluctuating asymmetry, trait variability, trait heritability, and stress: A multiply replicated experiment on combined stresses in Drosophila melanogaster. Evolution. 1999; 53:493-505. 

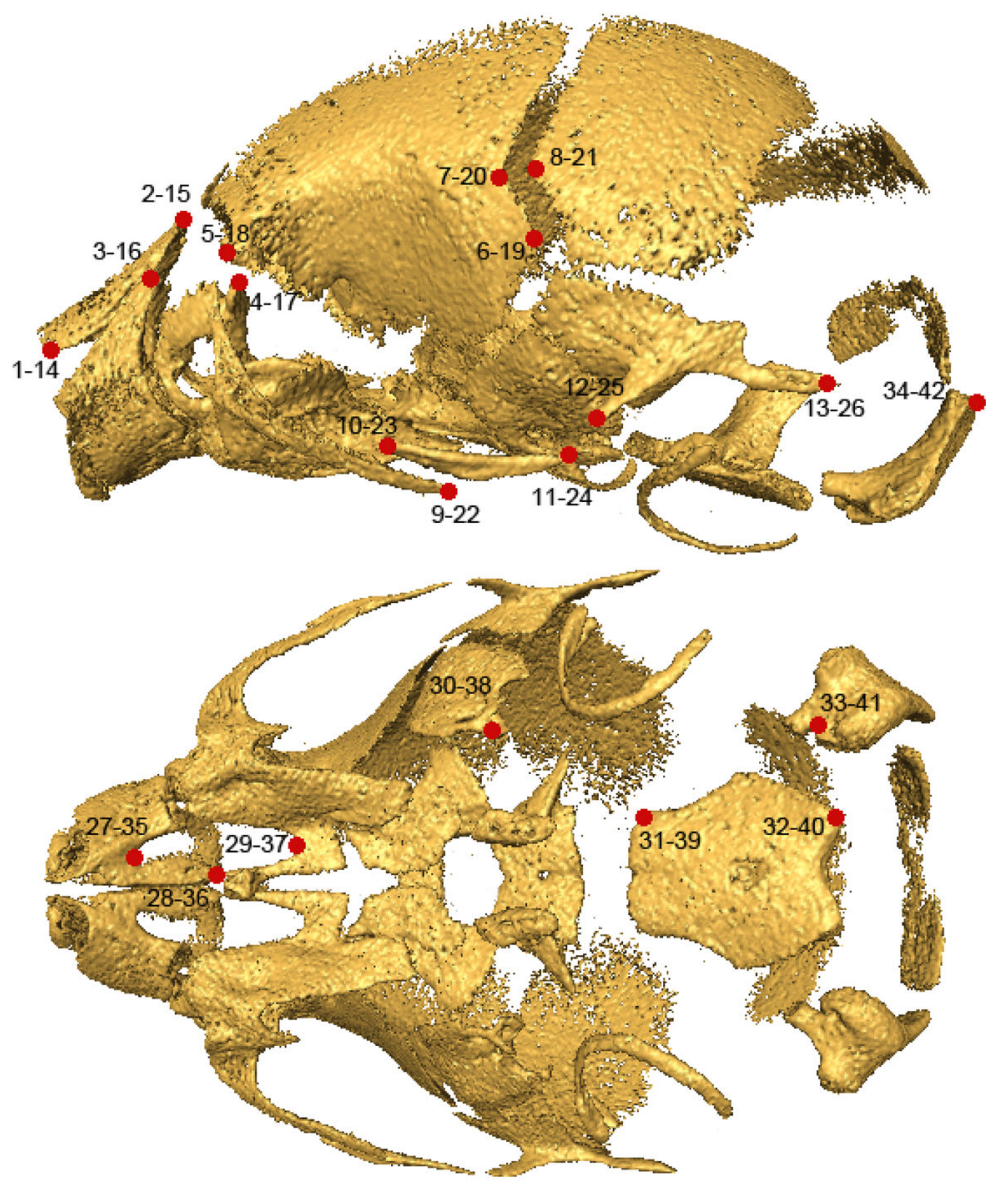

Fig. 1.

Coordinates of landmarks used to describe size and shape of fetal skull. 


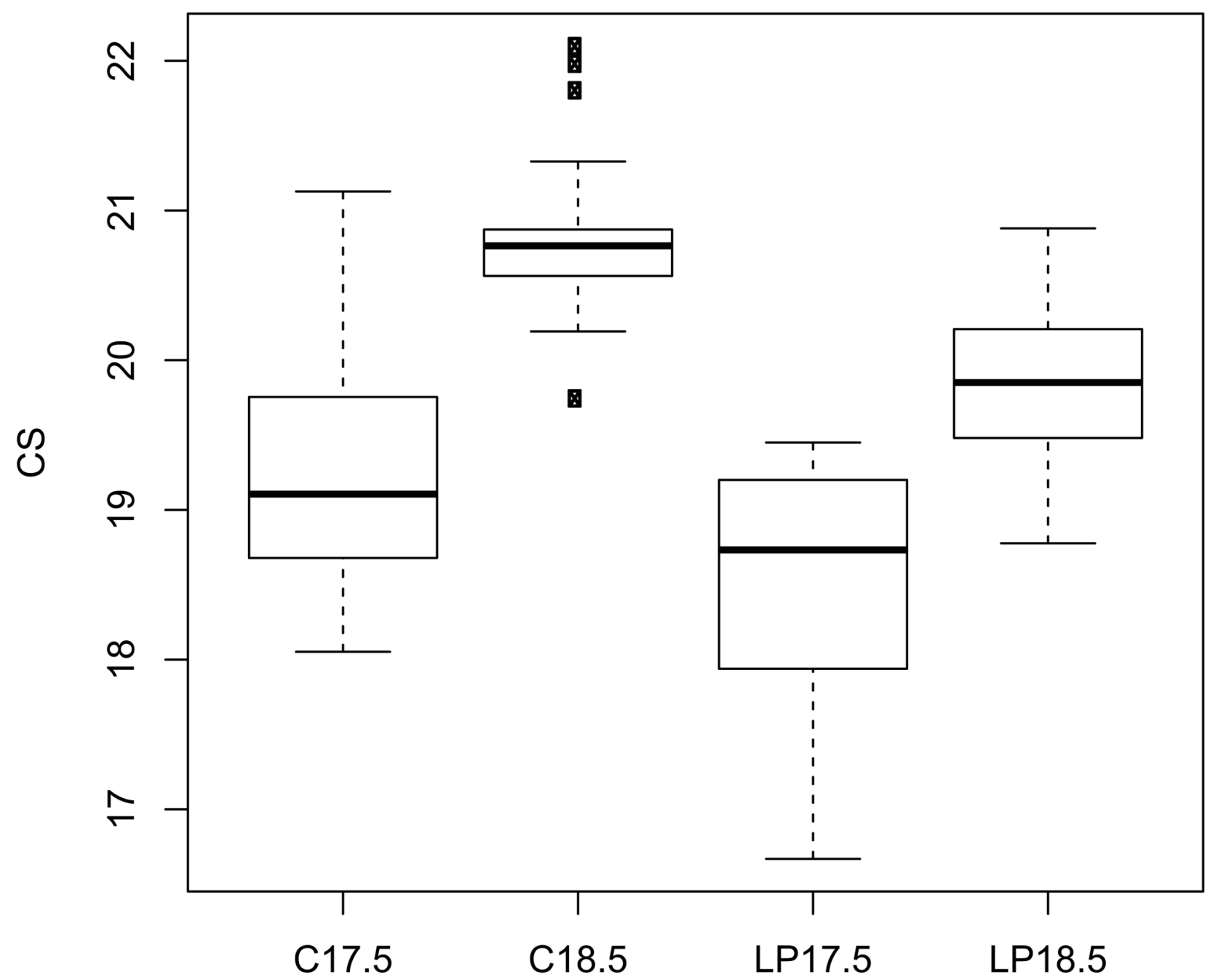

Fig. 2.

Centroid size of the skull in low protein (LP) and control (C) groups at the two prenatal stages studied (E17.5 and E18.5). 


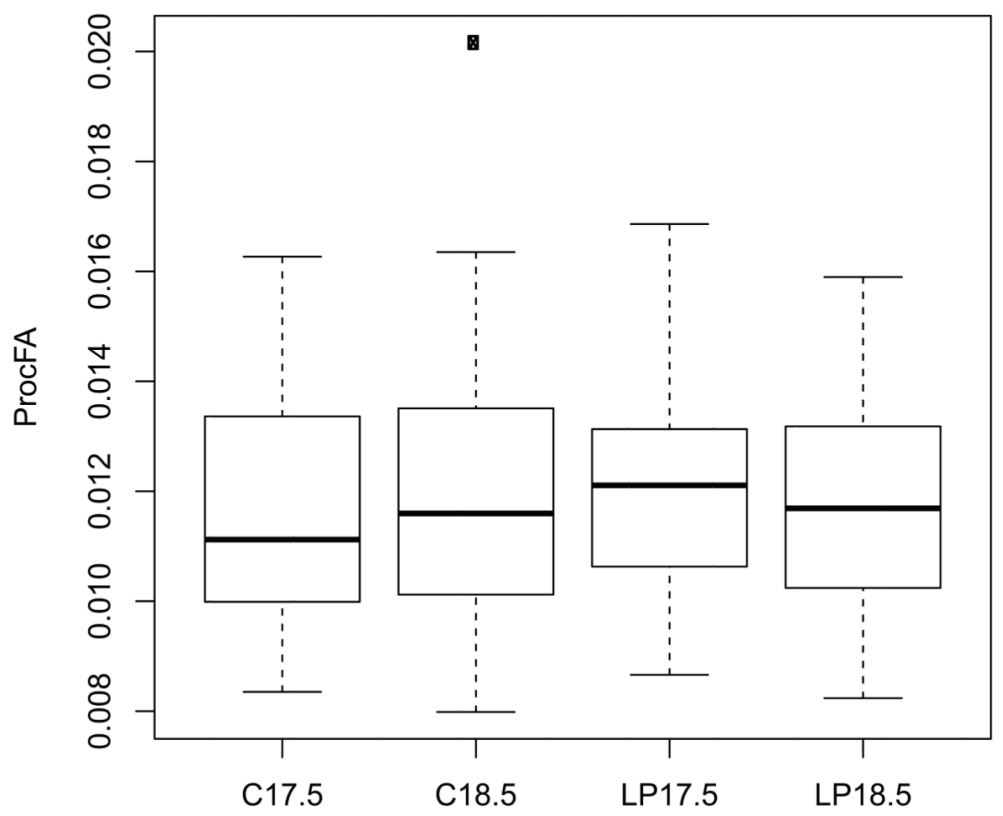

Fig. 3.

Procrustes scores of fluctuating asymmetry in low protein (LP) and control (C) groups at the two prenatal stages studied (E17.5 and E18.5). 


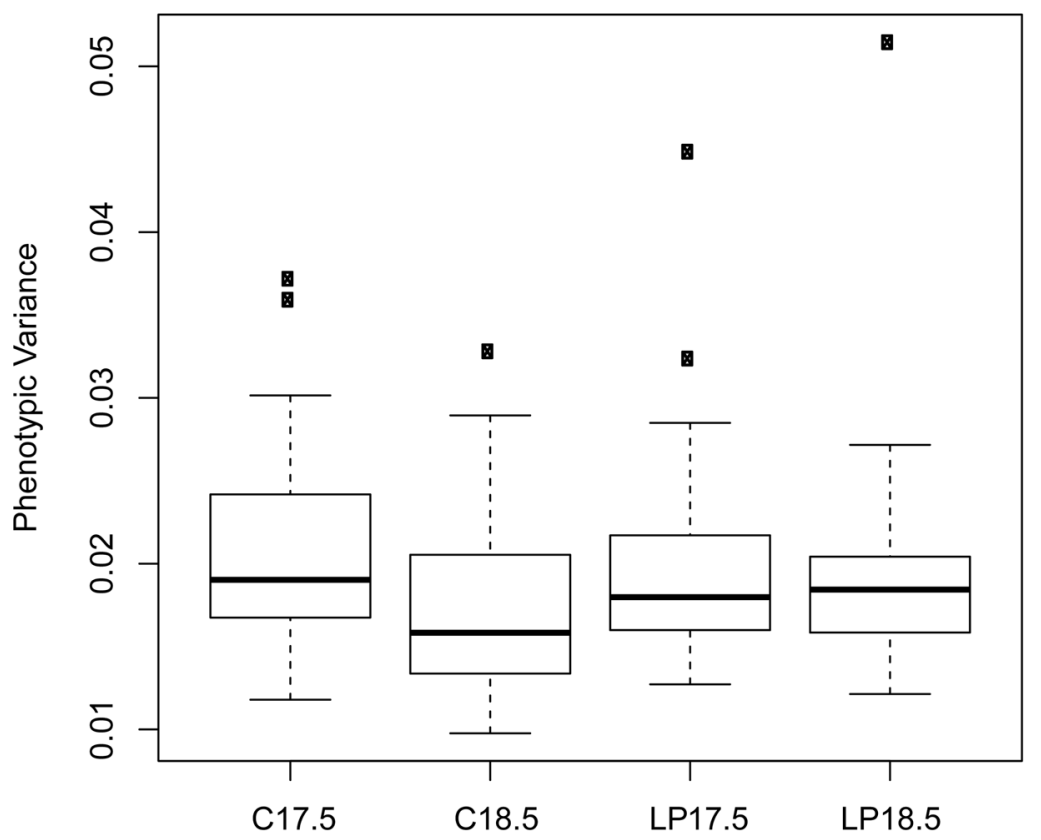

Fig. 4.

Shape variation within groups measured as the Procrustes distance of each specimen to its group mean. Low protein (LP) and control (C) at 17.5 and 18.5 days post-conception. 

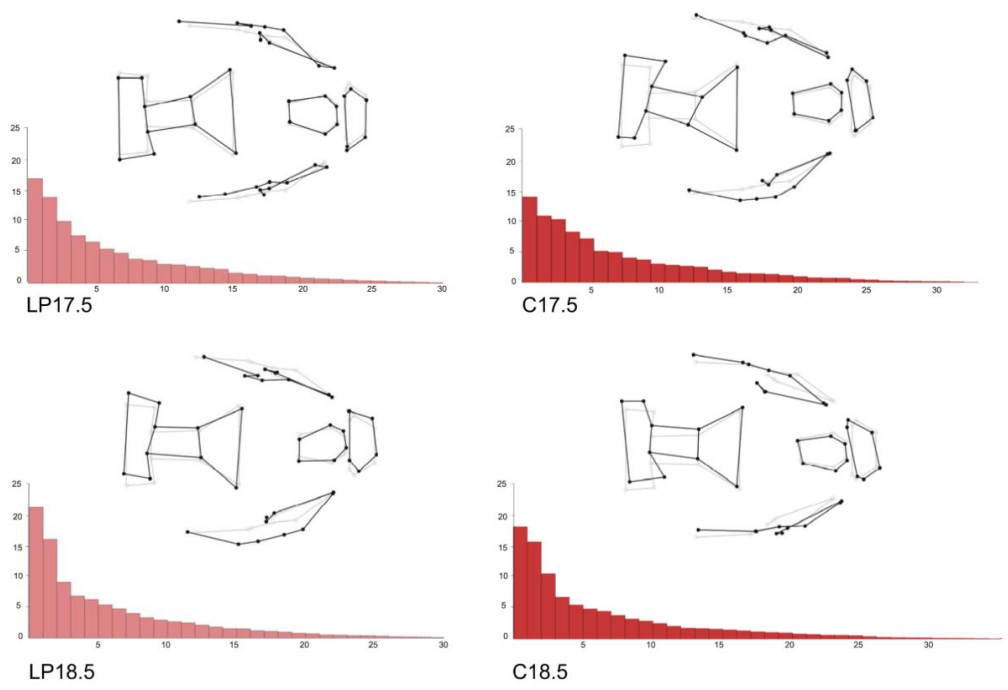

Fig. 5.

Distribution of eigenvalues from the principal component analysis of the asymmetric component of cranial shape in low protein (LP) and control (C) groups at 17.5 and 18.5 days post-conception. Wireframes depict the asymmetric shape variation from the mean (light gray) to the positive extreme of the first principal component (dark gray) for each group. 


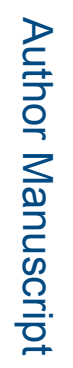

I

\begin{tabular}{|c|c|c|c|c|c|c|c|c|c|c|c|c|c|c|c|c|c|}
\hline 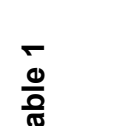 & 旁 & 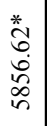 & $\stackrel{*}{\stackrel{*}{\sigma}}$ & 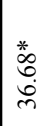 & & 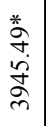 & $\begin{array}{l}* \\
\infty \\
\infty \\
\infty \\
\infty \\
\infty \\
\end{array}$ & $\begin{array}{l}* \\
\stackrel{*}{\circ} \\
\stackrel{\sim}{\dot{d}}\end{array}$ & & 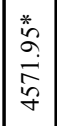 & 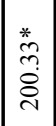 & 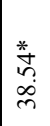 & & 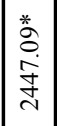 & 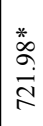 & $\stackrel{*}{\stackrel{*}{c}}$ & \\
\hline 80 & $\begin{array}{l}\bar{E} \\
\bar{\omega} \\
\bar{E} \\
\frac{\tilde{g}}{2}\end{array}$ & 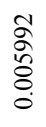 & $\begin{array}{l}\text { : } \\
8 \\
8 \\
8 \\
0\end{array}$ & 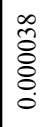 & 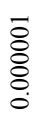 & 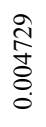 & 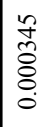 & 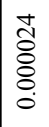 & $\begin{array}{l}\vec{\Xi} \\
\Xi \\
\vdots \\
\circ\end{array}$ & $\mid \begin{array}{l}\tilde{\delta} \\
\tilde{\sigma} \\
\delta \\
0 \\
0\end{array}$ & 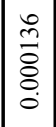 & 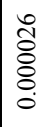 & $\begin{array}{l}\vec{\Xi} \\
\Xi \\
\vdots \\
\circ\end{array}$ & 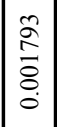 & $\begin{array}{l}\text { ते } \\
\text { ठे } \\
\vdots \\
0\end{array}$ & 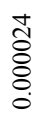 & छे \\
\hline
\end{tabular}

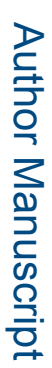

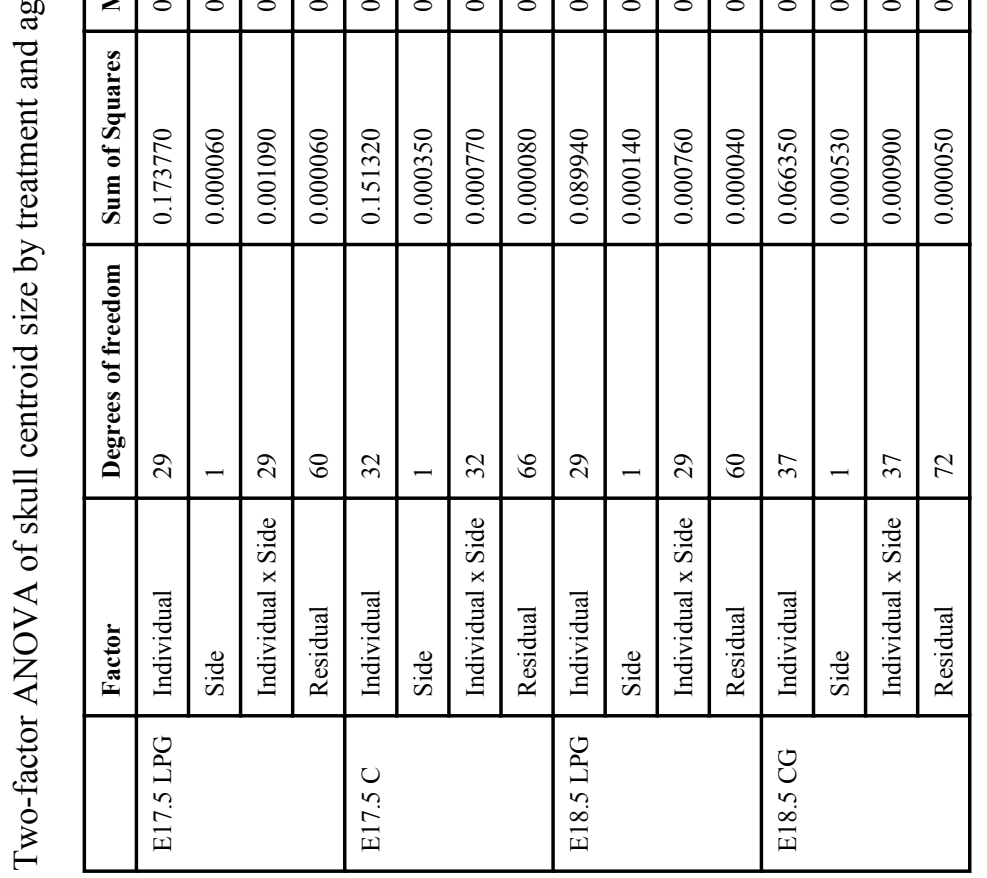

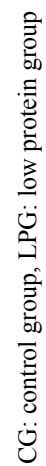

Am J Phys Anthropol. Author manuscript; available in PMC 2015 May 08. 
Table 2

Directional (DA) and fluctuating asymmetry (FA) of centroid size.

\begin{tabular}{|l|l|l|l|}
\hline Group & DA $=(\mathbf{L}-\mathbf{R})$ & $\mathbf{F A}=|(\mathbf{L}-\mathbf{R})-\mathbf{D A}|$ & FA10 \\
\hline E17.5 LPG & 0.0015 & 0.0048 & 0.0048 \\
\hline E17.5 CG & 0.0032 & 0.0039 & 0.0038 \\
\hline E18.5 LPG & 0.0021 & 0.0042 & 0.0040 \\
\hline E18.5 CG & 0.0034 & 0.0032 & 0.0038 \\
\hline
\end{tabular}

$\mathrm{L}=\mathrm{left}$ and $\mathrm{R}=$ right sides. FA10 index was estimated based on the values in Table 1 . 


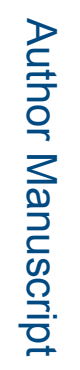

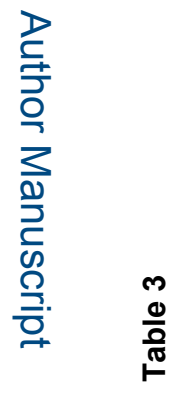

를

\begin{tabular}{|c|c|c|c|c|c|c|c|c|c|c|c|c|c|c|c|}
\hline & 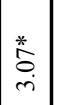 & $\stackrel{*}{\stackrel{*}{\sim}}$ & $\begin{array}{l}\stackrel{*}{\vec{a}} \\
\stackrel{\mathrm{I}}{\mathrm{I}}\end{array}$ & & 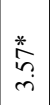 & ڤે & 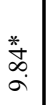 & & $\underset{\sim}{\stackrel{*}{二}}$ & $\begin{array}{l}* \\
\stackrel{*}{0} \\
\tilde{n} \\
\end{array}$ & 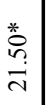 & & $\begin{array}{l}\stackrel{*}{\mathrm{~N}} \\
\underset{\mathrm{N}}{ }\end{array}$ & 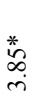 & \\
\hline & 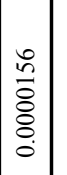 & $\begin{array}{l}3 \\
\overline{0} \\
\vdots \\
0 \\
0\end{array}$ & $\begin{array}{l}\overrightarrow{\tilde{n}} \\
0 \\
0 \\
0 \\
0\end{array}$ & 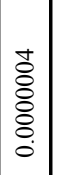 & 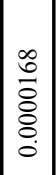 & 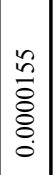 & 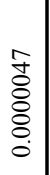 & ڤ气 & 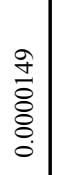 & 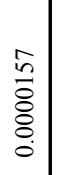 & $\begin{array}{l}\hat{y} \\
0 \\
0 \\
0 \\
0\end{array}$ & 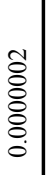 & $\begin{array}{l}\infty \\
0 \\
0 \\
\vdots \\
0 \\
0\end{array}$ & 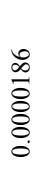 & 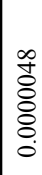 \\
\hline
\end{tabular}

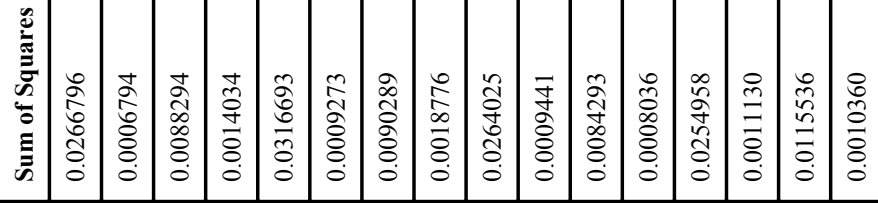

I

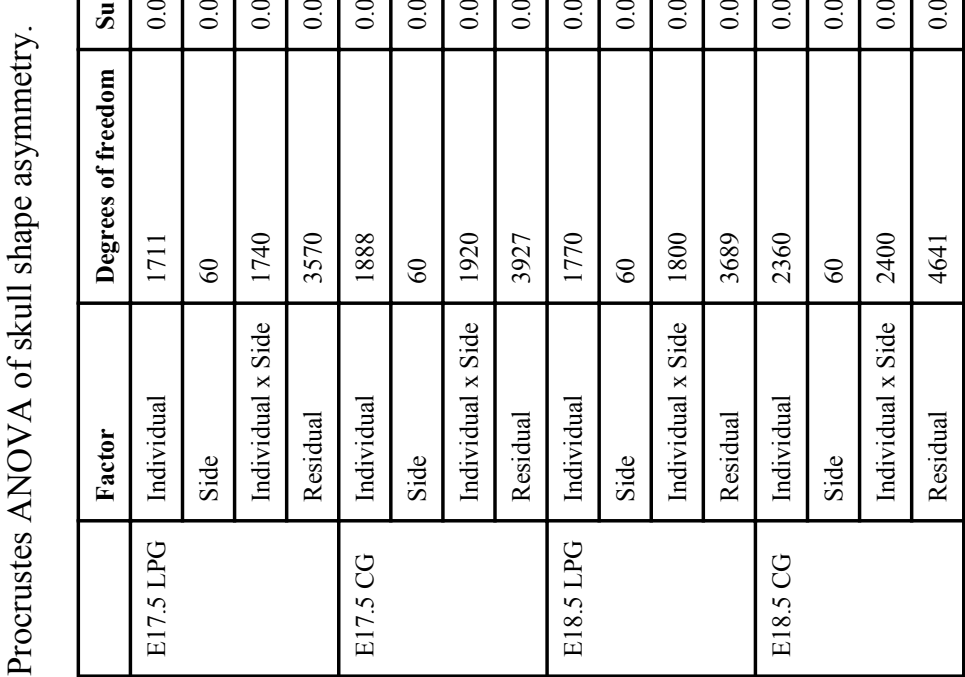

Am J Phys Anthropol. Author manuscript; available in PMC 2015 May 08. 


\section{Table 4}

Angles between first principal components (PC1) of each treatment for the asymmetric and symmetric components of shape.

\begin{tabular}{|l|l|l|}
\hline Age & Shape component & Angle between PC1 \\
\hline \multirow{2}{*}{ E 18.5 } & Asymmetric & $\begin{array}{l}84.185 \\
\mathrm{P}=0.43716\end{array}$ \\
\cline { 2 - 3 } & Symmetric & $\begin{array}{l}27.375 \\
\mathrm{P}<.00001\end{array}$ \\
\hline \multirow{2}{*}{ E 17.5 } & Asymmetric & $\begin{array}{l}72.659 \\
\mathrm{P}=0.09161\end{array}$ \\
\cline { 3 - 3 } & & 37.446 \\
& Symmetric & $\mathrm{P}<.00001$ \\
\hline
\end{tabular}

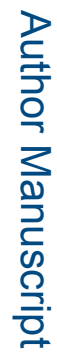

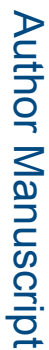

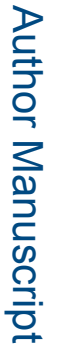

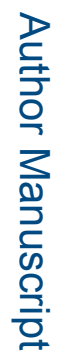

